\title{
Carbohydrate deficient serum transferrin in a new systemic hereditary syndrome
}

\author{
H Stibler, J Jaeken
}

\begin{abstract}
Four patients with a new, inherited, complex developmental deficiency syndrome were studied. The syndrome affects the central and peripheral nervous system, and also the retina, liver, bone, adipose tissue, and genital organs. Abnormalities of glycoproteins, glycopeptide hormones, and lipids have been found in serum from these patients, the most pronounced being increased cathodal forms of transferrin. Isoforms of serum transferrin were therefore analysed qualitatively and quantitatively by isoelectric focusing and isocratic anion exchange chromatography, and the carbohydrate composition was determined in transferrin isolated by immune affinity chromatography. All the patients had about tenfold raised serum concentrations of isotransferrins with higher isoelectric points than normal. Similar findings, though less pronounced, were made in all the fathers and in one of the mothers. Half the transferrin in the patients was constantly present in two principal abnormal cathodal forms in approximately equal amounts. Carbohydrate determinations in purified transferrin showed quantitatively similar deficiencies of sialic acid, galactose, and $\mathbf{N}$-acetylglucosamine, the mannose content being normal. The results suggest that either two or all of the normally four terminal trisaccharides in transferrin may be missing. A defect in the synthesis or catabolism, or both, of this trisaccharide, which is common to many secretory glycoconjugates, is likely. Apart from providing a quantitative diagnostic method, the present findings may serve as a basis for further studies of the metabolic deficiency in this syndrome.
\end{abstract}

A new hereditary disorder has recently been identified in four girls with severe neurological symptoms associated with complex biochemical abnormalities. ${ }^{12}$ The neurological syndrome includes psychomotor retardation, pronounced cerebellar hypoplasia with corresponding cerebellar dysfunction, alternating internal strabismus, and peripheral sensorimotor neuropathy. ${ }^{12}$ Convulsions and stroke like episodes have occurred in some patients. ${ }^{2}$ Other organs are variably affected, including retinitis pigmentosa, skeletal abnormalities, lipodystrophy and fat accumulations on the buttocks, hypertrophy of the labia majora, and retracted nipples. ${ }^{12}$ In all four patients there was variable hepatomegaly with distension of the endo- plasmic reticulum and Golgi apparatus, and lipid and membrane like inclusions in the lysosomes of hepatocytes. ${ }^{2}$

A number of abnormalities of serum glycoproteins, lipids, hormones, and enzymes have been found in these patients-for example, low concentrations of albumin, haptoglobin, apoprotein $\mathrm{B}$, thyroxine binding globulin, transcortin, and cholesterol in the serum, and fluctuating concentrations of prolactin, growth hormone, and follicle stimulating hormone. ${ }^{12}$ Some lysosomal enzyme activities in serum are raised, (arylsulphatase A, beta-galactosidase and $\mathrm{N}$-acetylglucosaminidase), and others are normal (sialidase and alpha-mannosidase). ${ }^{2}$ The microheterogeneity of transferrin from serum and cerebrospinal fluid was abnormal in all the patients, suggesting a deficiency in the negatively charged carbohydrate sialic acid. ${ }^{1}$ A similar abnormality was also found in other serum glycoproteins, but to a lesser extent. ${ }^{2}$

Studies of the carbohydrate content of total serum glycoproteins indicated that the carbohydrate deficiency was complex and included a reduction of about $40 \%$ not only of sialic acid, but also of galactose and $\mathrm{N}$-acetylglucosamine in the patients and to a lesser degree in their fathers as well. ${ }^{12}$ The carbohydrate content of total serum glycoproteins is, however, affected by the concentrations of the various glycoproteins, which also differ in their individual carbohydrate content and composition. In order to obtain more detailed information on this carbohydrate deficiency in a single quantitatively important glycoprotein, serum transferrin isoforms were studied qualitatively and quantitatively, and the carbohydrate content was determined in the isolated glycoprotein.

Subjects and methods

For analysis of carbohydrate concentrations in transferrin, serum was prepared from venous blood from the four female patients aged 2 $5-12$ years, ${ }^{12}$ and from four age matched controls. The patients, including monozygous twins, came from three unrelated families (table 1). For qualitative and quantitative analyses of isoforms of serum transferrin, samples were collected from six control children aged 2-14 years, from two clinically unaffected siblings aged $3 \cdot 5-4$ years, and from the three clinically healthy fathers and mothers. There was no history of alcohol abuse in the parents, and they had agreed not to use any alcohol for the two weeks preceding sampling. ${ }^{3}$

Serum samples were immediately frozen and 
Table 1. Summary of principal clinical symptoms shown by the patients

Growth mostly at or below the third centile

Psychomotor retardation without regression

Alternating internal strabismus

Hypotonia, trunk ataxia, hyporeflexia

Localised fat accumulations, lipodystrophy, obesity

Prominent hccumulations,

Variable hepatomegaly

Variable hepatomegaly

Unexplained fits with coma, convulsions, and hemiplegia

stored at $-80^{\circ} \mathrm{C}$ until analysed. The samples were transported without thawing in dry ice from Belgium to Sweden within 24 hours.

ISOELECTRIC FOCUSING

A $40 \mu \mathrm{l}$ sample of whole serum diluted 1:75 in distilled water was analysed from each subject in thin layer polyacrylamide gel in a $\mathrm{pH}$ gradient ranging from $2 \cdot 2$ to $11^{4}$ and stained by silver staining. ${ }^{5}$ Transferrin components were identified by subsequent immunofixation of serum samples containing a constant amount of transferrin $(0.5 \mu \mathrm{g})$ using antibodies against human serum transferrin (DAKO). ${ }^{6}$ Samples containing $25 \mu \mathrm{g}$ of iron saturated purified transferrin from each subject were examined and stained with Coomassie brilliant blue. Densitometry was then carried out and the relative amount of each component was calculated as a percentage of the total. ${ }^{7}$ Genetic typing of serum transferrin was carried out in the patients after neuraminidase treatment of serum, isoelectric focusing, and immunofixation using a sample with a known phenotype of C 1-2 as reference. ${ }^{8}$ Total transferrin concentration in the serum and in the samples with purified transferrin were determined by single radial immunodiffusion on NOR-Partigen plates (Behring).

\section{DETERMINATION OF CARBOHYDRATE DEFICIENT} TRANSFERRIN (CDT) IN SERUM

After iron saturation and dilution, duplicate samples of $100 \mu \mathrm{l}$ of serum were subjected to isocratic microanion exchange chromatography at pH 5.65 after which a transferrin radioimmune assay of the eluate was carried out as previously described. ${ }^{3}$ This method, initially developed for diagnosing alcohol abuse, measures the concentration of cathodal isotransferrins in serum (isoelectric point $\geqslant 5.7$ ) while the normal main components (isoelectric point $<5 \cdot 7)$ are retained by the anion exchanger. ${ }^{3}$ Except when rare genetic D variants of transferrin are present, ${ }^{9}$ increased concentrations of carbohydrate deficient transferrin $(>74 \mathrm{mg} / \mathrm{l})$ indicate a reduction in the content of the charged carbohydrate sialic acid in transferrin. ${ }^{3}$ Variations in the content of neutral carbohydrate in transferrin (galactose, N-acetylglucosamine, and mannose) can probably not be detected by this method. ${ }^{10}$ The specificity of this assay for detecting alcohol abuse is $99 \%$ in large clinical studies in adults. ${ }^{311} 12$

PURIFICATION AND CARBOHYDRATE

DETERMINATIONS OF SERUM TRANSFERRIN

Samples of $2.5 \mathrm{ml}$ of serum from patients and controls were diluted $1: 1$ in $0.05 \mathrm{M}$ sodium orthophosphate and $0.02 \mathrm{M}$ sodium citrate at $\mathrm{pH} 7 \cdot 2$, and applied to $1.2 \mathrm{ml}$ columns of antitransferrin-Sepharose 4B. Chromatography was carried out as previously described. ${ }^{13} 14$ Two columns were used for each individual. The eluted transferrin from each subject was pooled, neutralised, dialysed, and concentrated to a final transferrin concentration of $1 \cdot 56-5 \cdot 34$ $\mathrm{g} / \mathrm{l}$ as determined by single radial immunodiffusion. ${ }^{14}$ Aliquots were saturated with iron and subjected to isoelectric focusing while the remainder of the concentrated eluates was used for carbohydrate analyses. ${ }^{14}$

The amounts of sialic acid, galactose, $\mathrm{N}$ acetylglucosamine, and mannose were determined after acid hydrolysis by colorimetric ${ }^{15} 16$ or enzymatic ${ }^{17}$ methods previously described, ${ }^{14}$ with the exception that the galactose content was analysed after hydrolysis in $0.5 \mathrm{M}$ sulphuric acid at $100^{\circ} \mathrm{C}$ for four hours, which had been found to improve reproducibility ( $H$ Stibler, unpublished observations). All samples were analysed for the same carbohydrate simultaneously on the same day and at least two specimens were examined. The variation of these methods (SD), varied between 0.07 and $4.7 \mathrm{nmol}^{14}$

\section{Results}

In the type of $\mathrm{pH}$ gradient used for isoelectric focusing, normal, iron saturated serum transferrin separates into six components within the $\mathrm{pH}$ range $5 \cdot 2-5 \cdot 7 .{ }^{4}$ The normal main component has an isoelectric point of 5.4 and corresponds to transferrin containing four sialic acid residues in its two carbohydrate chains (tetrasialotransferrin). ${ }^{4} 101418$ Anodal and cathodal isotransferrins are normally minor bands: higher glycosylated forms with isoelectric points of $5 \cdot 2$ and 5.3 , and trisialotransferrin and disialotransferrin with isoelectric points to 5.6 and $5 \cdot 7$, respectively. ${ }^{4101418}$ In all the patients there was a pronounced abnormality of the microheterogeneity of transferrin in native serum as well as in transferrin purified by affinity chromatography (figs 1 and 2). There was a pronounced increase (see below) of components with isoelectric points of 5.7 and 5.9 (disialotransferrin and asialotransferrin). Only a minor band at isoelectric point $5 \cdot 8$ (monosialotransferrin) was present, while components corresponding to trisialotransferrin, tetrasialotransferrin, and pentasialotransferrin (isoelectric points $5 \cdot 6$, $5 \cdot 4$, and $5 \cdot 3$, respectively) were less marked than normal. Moderate increases of disialotransferrin and asialotransferrin were observed in serum from all the fathers (fig 1). Neuraminidase treatment completely abolished the abnormal transferrin heterogeneity, and transferrin phenotypes were the common $\mathrm{Cl}$ in three patients, and $\mathrm{Cl}-2$ in one patient.

Quantitative determination of carbohydrate deficient transferrin (isoelectric point $>5.65$ ) showed approximately a tenfold rise in cathodal isotransferrins in all the patients compared with normal values, ${ }^{3} 912$ The carbohydrate deficient transferrin values were on average twice the normal in all three fathers, and slightly higher than normal in one of the mothers $(86 \mathrm{mg} / \mathrm{l})$. 


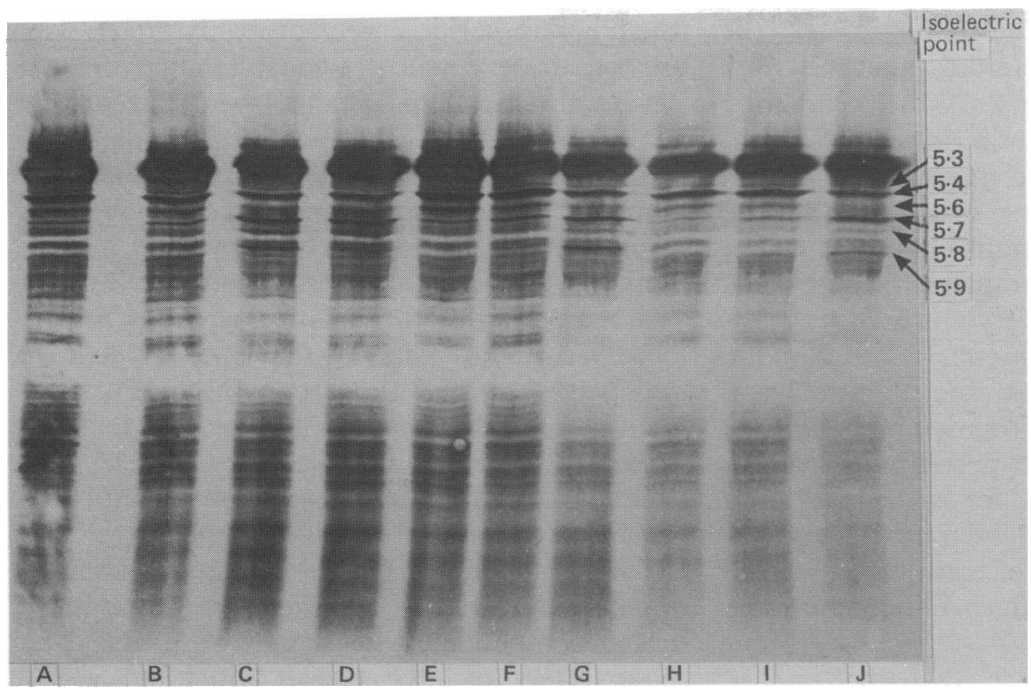

Figure 1 Isoelectric focusing of native serum proteins with silver staining within the $p H$ range 2:2-11. The isoelectric points of transferrin are indicated at the right. $A=$ mother of $C$ and $D . B=$ father of $C$ and $D$. $C$ and $D=$ patients. $E=$ mother of $G . F=$ father of $G$. $G=$ patient. $H=$ mother of $\mathcal{F} . I=$ father of $\mathcal{F} . \mathcal{F}=$ patient. Note the increase of disialotransferrin and asialotransferrin (isoelectric points $5 \cdot 7$ and $5 \cdot 9$ ) and the low amount of trisialotransferrin and monosialotransferrin (isoelectric point 5.6 and 5.8 ) in the patients. Note also the slight increase of disialotransferrin in the fathers. The transferrin pattern seems normal in the mothers.

Figure 2 Isoelectric focusing (as in fig 1) of $25 \mu \mathrm{g}$ of serum transferrin isolated by affinity chromatography and stained with Coomassie brilliant blue. The isoelectric points indicate transferrin components. A to $D=$ transferrin from controls, $E$ to $H=$ trainsferrin from the four patients. Note the preserved pronounced abnormality in transferrin from the patients. The most anodal band in some samples is contaminating albumin. The poorly focused material just cathodal to isoelectric point $5 \cdot 7$ is lipid.

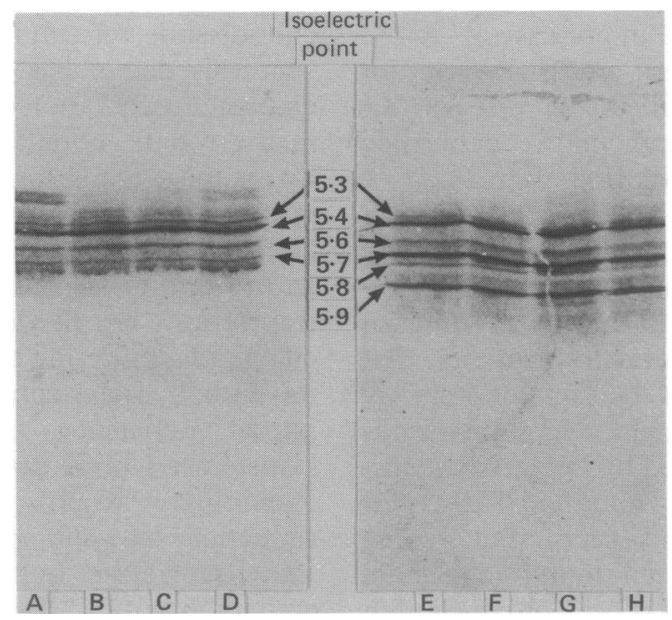

The other two mothers and the siblings had normal carbohydrate deficient transferrin concentrations (table 2). For the sake of comparison, carbohydrate deficient transferrin values from alcoholic patients are included in table 2 . Total transferrin concentration in serum was reduced $(1.40$ and $1.56 \mathrm{~g} / \mathrm{l})$ in two of the patients, but normal in the other two cases $(2.05$ and $2.94 \mathrm{~g} / \mathrm{l})$ (normal range $2 \cdot 02-3 \cdot 90 \mathrm{~g} / \mathrm{l}$ ).

Table 2 Concentrations of carbohydrate deficient transferrin in serum of patients, family members, and controls

\begin{tabular}{lc}
\hline Subjects & $\begin{array}{l}\text { Mean }(S D) \text { carbohydrate } \\
\text { deficient transferrin } \\
\text { concentration }(m g / l)\end{array}$ \\
\hline Patients $(\mathrm{n}=4)$ & $591(77)$ \\
Siblings $(\mathrm{n}=2)$ & $63(2)$ \\
Child controls $(\mathrm{n}=6)$ & $62(18)$ \\
Fathers $(\mathrm{n}=3)$ & $125(50)$ \\
Mothers $(\mathrm{n}=3)$ & $64(15)$ \\
Healthy adult controls $(\mathrm{n}=155)^{3}$ & $53(11)$ \\
Alcoholic patients $(\mathrm{n}=100)^{3}$ & $133(57)$ \\
\hline
\end{tabular}

Isoelectric focusing of immune affinity purified transferrin showed a preserved transferrin pattern and contamination with only traces of albumin (isoelectric point $4 \cdot 8-4 \cdot 9$ ) and lipid (isoelectric point $>5 \cdot 7$ ) (fig 2), as has been found before with this technique. ${ }^{14}$ As these contaminants contain no carbohydrate, they were not considered to affect the carbohydrate analyses.

In the patients, the percentage (SD) amounts of isotransferrins were $29(4) \%$ for the component with an isoelectric point of 5.7 (normal $\left.3.7(1.5) \%^{7}\right)$, and $24(3) \%$ for the one with an isoelectric point of 5.9 (normal 0 )\%. Normal isoforms (isoelectric point $5 \cdot 2-5 \cdot 6$ ) constituted 47 (3)\% of the total amount. The carbohydrate values in control transferrin corresponded closely to those accepted for normal whole transferrin, and to values obtained by the same methods in adults (table 3). ${ }^{1014}$ Compared with healthy adults ${ }^{14}$ the values in child controls tended to be somewhat higher, especially of $\mathrm{N}$ acetylglycosamine $(+13 \%)$, which might either be analytical or indicate a slightly higher degree of transferrin glycosylation in children. In all the patients, the total contents of sialic acid, galactose, and $\mathrm{N}$-acetylglucosamine in transferrin were much lower than in the control children. The average reduction was 31 (2)\% for all three carbohydrates. The mannose content, on the other hand, was almost identical in the two groups (table 3 ). Together, carbohydrate deficient isotransferrins (isoelectric point $5 \cdot 7-5 \cdot 9)$ constituted $53(2) \%$ of the isolated

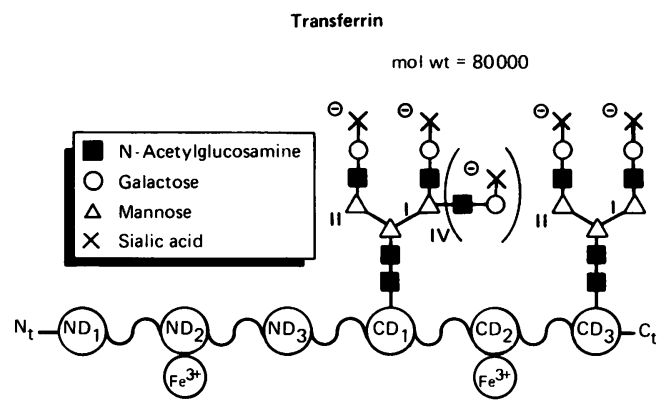

Figure 3 Oligosaccharide units of the main forms of normal transferrin. The trisaccharide in parentheses is normally present in only some of the molecules. ${ }^{10}$ The arrows indicate the sites of action of $I=N$-acetylglucosaminyltransferase $I$, which is essential for the action of

$I I=N$-acetylglycosaminyltransferase $I I$ and

$I V=N$-acetylglucosaminyltransferase $I V .^{25}$

Table 3 Carbohydrate concentrations in purified whole serum transferrin from patients and controls

\begin{tabular}{lcc}
\hline Carbohydrate & Patients & Controls \\
\hline Sialic acid: & & \\
Transferrin $(\mu \mathrm{mol} / \mathrm{g})$ & $37 \cdot 40(2 \cdot 07)$ & $54 \cdot 59(2 \cdot 27)$ \\
Transferrin $(\mathrm{mol} / \mathrm{mol})$ & $2 \cdot 8(0 \cdot 2)$ & $4 \cdot 2(0 \cdot 2)$ \\
& & \\
Galactose: & $38 \cdot 73(2 \cdot 58)$ & $54 \cdot 71(1 \cdot 38)$ \\
Transferrin $(\mu \mathrm{mol} / \mathrm{g})$ & $4 \cdot 2(0 \cdot 1)$ \\
Transferrin $(\mathrm{mol} / \mathrm{mol})$ & $3 \cdot 0(0 \cdot 2)$ & \\
N-acetylglucosamine: & & $118 \cdot 33(5 \cdot 06)$ \\
Transferrin $(\mu \mathrm{mol} / \mathrm{g})$ & $83 \cdot 38(4 \cdot 36)$ & $9 \cdot 1(0 \cdot 4)$ \\
Transferrin $(\mathrm{mol} / \mathrm{mol})$ & $6 \cdot 4(0 \cdot 3)$ & \\
Mannose: & & $78 \cdot 85(5 \cdot 43)$ \\
Transferrin $(\mu \mathrm{mol} / \mathrm{g})$ & $76 \cdot 05(1 \cdot 82)$ & $6 \cdot 1(0 \cdot 4)$ \\
Transferrin $(\mathrm{mol} / \mathrm{mol})$ & $5 \cdot 9(0 \cdot 1)$ & \\
\hline
\end{tabular}

The molar ratios were calculated from a molecular weight of 77000 for transferrin. 
glycoprotein. The average reduction of the three saccharides may therefore amount to $66 \%$ in the abnormal fractions. As these mainly consisted of disialotransferrin (29\%) and asialotransferrin $(24 \%)$, and the three carbohydrates were reduced to the same extent, it may be suggested that the disialotransferrin component lacks two, and the asialotransferrin four, sialic acid, galactose, and $\mathrm{N}$-acetylglucosamine residues peripheral to mannose (fig 3). Based on the relative amounts of normal and abnormal isotransferrins, this type of proposed carbohydrate defect would result in expected total carbohydrate contents of 2.6 for sialic acid and galactose, 6.9 for $\mathrm{N}$-acetylglucosamine, and $6 \cdot 1$ for mannose (in $\mathrm{mol} / \mathrm{mol}$ of transferrin), which are close to the total values obtained in the patients (table 3 ).

\section{Discussion}

The extreme alteration of transferrin microheterogeneity on isoelectric focusing, with more than half of this glycoprotein being abnormal in our patients, seems to be almost specific for this disease. An abnormality of this kind has also been described recently in seven Swedish children (four girls and three boys from four families) with a constellation of complex clinical symptoms similar to those in our cases. ${ }^{19}$ Based on the transferrin pattern on isoelectric focusing, these authors called the disease 'disialotransferrin developmental deficiency syndrome'. ${ }^{19}$ From their first figure it is, however, clear that both the disialotransferrin and asialotransferrin components were greatly increased in serum as in our patients. The clinical picture, together with the most unusual appearance of the transferrin, suggests that these patients suffer from the same or a closely related metabolic disorder. In several thousands of serum samples examined by isoelectric focusing by us and others ${ }^{19} 20$ a cathodal distribution of transferrin of this type, though less pronounced, has only been found in alcohol abusers. The same result has been obtained with the more rapid and simple quantitative carbohydrate deficient transferrin assay. ${ }^{3} 9111221$ Slightly increased carbohydrate deficient transferrin concentrations may occur in some patients with primary biliary cirrhosis, and in patients with rare genetic D-variants of transferrin. ${ }^{9} 1112$ Carbohydrate deficient transferrin concentrations of the magnitude of those in the present cases, however, have only occasionally been found in alcoholics. With these exceptions in mind, the carbohydrate deficient transferrin assay seems to be highly specific for this syndrome.

The results of carbohydrate analyses in total serum glycoproteins ${ }^{12}$, and now specifically in isolated transferrin, clearly show that the carbohydrate deficiency includes not only the charged sialic acid but also the neutral galactose and $\mathrm{N}$-acetylglucosamine to almost exactly the same extent, which together constitute the terminal trisaccharides in transferrin (fig 3 ) and in many other secretory glycoproteins. ${ }^{22}$ The normal mannose content and the degree of reduction of $\mathrm{N}$-acetylglucosamine suggest that the oligosaccharide core is probably intact. The carbohydrate defect previously shown in total serum glycoproteins ${ }^{12}$ cannot be quantitatively explained by this abnormality in transferrin alone. Other serum glycoproteins can therefore be expected to be similarly affected, and this supposition is also indirectly supported by preliminary isoelectric focusing analyses. ${ }^{2}$ One of the reasons why this change is more readily observed in transferrin may be its relative independence of the carbohydrate units for secretion and survival in circulation in contrast to many other serum glycoproteins. ${ }^{23} 24$

The fact that the transferrin abnormality consists of increases of disialotransferrin and asialotransferrin, and only to a small extent of monosialotransferrin together with the reduced trisialocomponent, argues against a simple catabolic effect of glycosidases. Such an action would be expected to cause a successive or random increase in cathodal isotransferrin, ${ }^{4} 18$ provided that the recognition and elimination of transferrin with two or four defective oligosaccharide antennas is not selectively impaired. ${ }^{23}$ The normal sialidase activity and normal concentrations of free sialic acid, galactose, and $\mathrm{N}$-acetylglucosamine in serum are also evidence against a glycolytic mechanism. ${ }^{2}$

In view of the morphological pathology of the endoplasmic reticulum and Golgi apparatus, a synthesis defect has to be considered. ${ }^{2}$ After en bloc incorporation of the core oligosaccharide in the rough endoplasmic reticulum, followed by mannose trimming, the carbohydrates of the terminal trisaccharides in serum glycoproteins are successively added in the Golgi complex. ${ }^{25}$ In the case of transferrin at least five glycosyltransferases are needed for completion of the chain. ${ }^{25}$ Considering the transferrin pattern and its carbohydrate contents in the patients, a partial deficiency of $\mathrm{N}$-acetylglucosaminyltransferase I or II can be hypothesised (fig 3). Preliminary results have indicated a $40 \%$ reduction of non-specified $\mathrm{N}$-acetylglucosaminyltransferase but also of sialyltransferase activity in serum. ${ }^{2}$ To elucidate the basic defect, studies of the intracellular enzymes with specific isoenzyme analyses are in progress. It may be of interest that in alcoholic patients with a carbohydrate deficiency in transferrin similar to that shown by the patients with this syndrome ${ }^{14}$ several glycosyltransferases have low activities, apparently as a result of an inhibitory action of acetaldehyde, ${ }^{26}$ which also causes morphological changes of the Golgi apparatus. ${ }^{27}$ The essential similarity in symptoms, particularly those of the nervous system, between this inherited syndrome and the acquired complications of chronic alcoholism in adults is notable. These two conditions may therefore offer ways to gain greater insight into the role of the carbohydrate moiety in secretory glycoproteins for the development and maintenance of the human nervous system.

We thank Dr M Joustra of Pharmacia, Uppsala, for supplying the antitansferin sepharose 4B, and Mrs Birgitta Cederberg, Mrs Marianne Gyllenpalm, and Mrs Ann-Marie Olsson for 
excellent technical assistance. This work was supported by grants from the Swedish Medical Research Council (grants no 07339-03 and 07212-03 B), the Karolinska Institute and the Vivian Smith Foundation for Restorative Neurology.

1 Jaeken J, Eggermont E, Stibler H. An apparent homozygous $\mathrm{X}$-linked disorder with carbohydrate-deficient serum glycoproteins. Lancet 1987;ii:1398.

2 Jaeken J, Stibler H. A newly recognized inherited neurological disease with carbohydrate-deficient secretory glycoproteins. In: Wetterberg L, ed. Genetics of neuropsychiatric diseases. Wenner-Gren International Symposium Series. Vol 51. London: Macmillan Press, 1989:69-80.

3 Stibler $\mathbf{H}$, Borg S, Joustra M. Micro anion exchange chromatography of carbohydrate-deficient transferrin in serum in relation to alcohol consumption. Alcoholism: Clinical and Experimental Research 1986;10:535-44.

4 Stibler $\mathrm{H}$. The normal cerebrospinal fluid proteins identified by means of isoelectric focusing and crossed immunoelectrofocusing. I Neurol Sci 1978;36:273-88.

5 Schipper H, Kruse H, Reiber H. Silver-staining of oligoclonal IgG subfractions in cerebrospinal fluid after isoelectric focusing in thin-layer polyacrylamide gels. Science Tools 1984;31:5-6.

6 Stibler H. Direct immunofixation after isoelectric focusing. I Neurol Sci 1979;42:275-81.

7 Stibler H, Sydow O, Borg S. Quantitative estimation of abnormal microheterogeneity of serum transferrin in alcoholics. Pharmacol Biochem Behav 1980;13:47-5

8 Stibler H, Beckman G, Sikstrom C. Subtypes of transferrin C. Hum Hered 1979;29:320-24.

9 Stibler H, Borg S, Beckman G. Transferrin phenotype and level of carbohydrate-deficient transferrin in healthy individuals. Alcoholism: Clinical and Experimental Research 1988;12:450-3.

10 Spik G, Bayard B, Strecker G, Bouquelet S, Montreuil J. Studies on glycoconjugates. LXIV. Complete structure of two carbohydrate units of human serotransferrin. FEBS Lett 1975;50:296-9.

11 Behrens U, Worner T, Braly L, Schaffner F, Lieber C. Carbohydrate-deficient transferrin, a marker for chronic alcohol consumption in different ethnic populations. Alcoholism: Clinical and Experimental Research 1988;12: 539-44.

12 Stibler H, Borg S, Joustra M, Hultcrantz R. Carbohydratedeficient transferrin (CDT) in serum as a marker of high deficient transferrin (CDT) in serum as a marker of high
alcohol consumption. In: Nordmann $R$, Ribière $C$
Rouach H, eds. Advances in the biosciences. Vol 71. Oxford: Pergamon Press, 1988:347-53.

13 van Eijk H, van Noort W. Isolation of rat transferrin using CNBr-activated Sepharose 4B. $\mathcal{F}$ Clin Chem Clin Biochem 1976;14:475-8.

14 Stibler H, Borg S. Carbohydrate composition of serum transferrin in alcoholic patients. Alcoholism: Clinical and Experimental Research 1986;10:61-4.

15 Elson L, Morgan W. A colorimetric method for the determination of glucosamine and chondrosamine. Biochem $\mathcal{J}$ 1933;27:1824-38.

16 Warren $L$. The thiobarbituric acid assay of sialic acids. 7 Biol Chem 1959;234:1971 5 .

17 Finch P, Yuen R, Schachter H, Moscarello M. Enzymic methods for the microassay of D-mannose, D-glucose, D-galactose and L-fucose from acid hydrolysates of glycoproteins. Anal Biochem 1969;31:296-305.

18 Hamann A. The microheterogeneity of serum glycoprotein as revealed by flat-bed isoelectric focusing. In: Radola BJ, Graesslin B, eds. Electrofocusing and isotachophoresis. New York: W deGryter, 1977:329-35.

19 Kristiansson B, Andersson M, Tonnby B, Hagberg B. The disialotransferrin developmental deficiency (DDD-) syndrome. Arch Dis Child 1989;64:71-6.

20 Stibler H, Borg S, Allgulander C. Clinical significance of abnormal heterogeneity of transferrin in relation to alcohol abnormal heterogeneity of transferrin in relation to
consumption. Acta Med Scand 1979;206:275-81.

21 Gjerde H, Johnsen J, Bjørneboe A, Biørneboe G-E, Mørland J. A comparison of serum carbohydrate-deficient trans-
ferrin with other markers of excessive drinking. Scand 7 Clin Lab Invest 1988;48: 1-6.

22 Montreuil J. Primary structure of glycoprotein glycans. Basis for the molecular biology of glycoproteins. Adv Carbohydr Chem Biochem 1980;37:157-223.

23 Ashwell G, Harford J. Carbohydrate-specific receptors of the liver. Annu Rev Biochem 1982;51:531-54.

24 Struck D, Siuta P, Lane D, Lennarz W. Effect of tunicamycin on the secretion of serum proteins by primary cultures of rat and chicken hepatocytes. F Biol Chem 1978;253:5332-7.

25 Schachter H. Biosynthetic controls that determine the branching and microheterogeneity of protein-bound oligosaccharides. Biochem Cell Biol 1985;64:161-81.

26 Stibler $\mathrm{H}$, Borg S. The value of carbohydrate-deficient transferrin as a marker of high alcohol consumption. In: Kuriyama $\mathrm{K}$, Takada A, Ishii $\mathrm{H}$, eds. Biomedical and social aspects of alcohol and alcoholism. Amsterdam: Elsevier, aspects of a $1988: 503-6$.

27 Matsuda Y, Baraona E, Salaspuro M, Lieber C. Effects of chronic ethanol administration on liver microtubules and Golgi apparatus. Lab Invest 1979;41:455-63. 